\title{
Correlation between APRI, FIB-4 and GPR Indices to Fibroscan and HBeAg Status in Patients with Chronic Hepatitis B
}

\author{
Rina Erlina ${ }^{1}$, Puspa Wardhani ${ }^{1}$, Yessy Puspitasari ${ }^{1}$, Ulfa Kholili ${ }^{2}$ \\ ${ }^{1}$ Department of Clinical Pathology, Faculty of Medicine, Airlangga University/Dr. Soetomo Hospital, Surabaya, Indonesia. E-mail: \\ drrina_05@yahoo.com \\ ${ }^{2}$ Department of Internal Medicine, Faculty of Medicine, Airlangga University/Dr. Soetomo Hospital, Surabaya, Indonesia
}

\begin{abstract}
Liver fibrosis is a complication of chronic hepatitis B. Early detection of liver fibrosis is important for therapy. The aspartate aminotransferase index (AST)-to-platelet ratio index (APRI) and the fibrosis index based on 4 factors (FIB-4) in chronic hepatitis $B$ have been widely studied despite the inconsistent results. Research on other serum markers is extensively carried out, including Gamma-Glutamyl Transpeptidase (GGT)-to-platelet ratio (GPR). Previous studies have shown that the GPR index was more accurate than APRI and FIB-4. HBeAg status is an indication for therapy. There have not been many studies on the correlation of serum markers with HBeAg status. This study aimed to determine the correlation of APRI, FIB-4, and GPR with Fibroscan and $\mathrm{HBeAg}$ status in chronic hepatitis B patients. A cross-sectional study was carried out from June to September 2020 and found 50 chronic hepatitis B patients. Platelet count was measured using a Sysmex XN-1000 hematology device; AST, alanine aminotransferase (ALT), and GGT levels were measured using the Dimension RXL clinical chemistry device; and the degree of fibrosis was determined using transient elastography (Fibroscan). Spearman correlation test was used in this study for the correlation analysis. The results showed a significant correlation between APRI, FIB-4 and GPR indices with Fibroscan ( $r=0.454, p 0.001 ; r=0.610, p<0.001 ; r=0.540, p<0.001$, respectively). A significant correlation was found between APRI, FIB-4 and GPR indices with negative $(-)$ HBeAg $(r=0.486, p$ 0.004; $r=0.648, p<0.001 ; r=0.595$, $\mathrm{p}<0.001$, respectively). In addition, a significant correlation was found between FIB-4 and positive $(+) \mathrm{HBeAg}(r=0.499$, p 0.049), but no correlation was found between APRI and GPR with positive (+) HBeAg $(r=0.295, p$ 0,267; $r=0.386, p$ 0.140, respectively).
\end{abstract}

Keywords: APRI, FIB 4, GPR, fibroscan, chronic hepatitis B, HBeAg

\section{INTRODUCTION}

Chronic hepatitis $B$ is a dynamic disease condition, which may develop into liver fibrosis, liver cirrhosis, and hepatocellular carcinoma. The decision to start therapy can reduce the risk of these complications. Early detection of liver fibrosis is important for determining therapy. Factors that influence treatment decisions include $\mathrm{HBeAg}$ status, ALT levels, HBV DNA, and histological tests to determine the severity of liver fibrosis. ${ }^{1}$

$\mathrm{HBeAg}$ is a marker of viral replication and the infectious level of chronic hepatitis B. ALT levels $>2 x$ the upper limit of normal and HBV DNA $>20,000$ $\mathrm{IU} / \mathrm{mL}$ on positive (+) $\mathrm{HBeAg}$ or $>2,000 \mathrm{IU} / \mathrm{mL}$ on negative (-) HBeAg are the recommended therapy. The treatment is determined by histological test on the degree of fibrosis if the patients have normal ALT levels and positive (+) or negative (-) HBeAg status. ${ }^{2}$

The gold standard for the determination of the degree of liver fibrosis is liver biopsy. Liver biopsy is an invasive procedure with complications of pain, bleeding, and discomfort for the patient. The accuracy of the liver biopsy results is determined by the quality of the tissue sample and expertise in the interpretation of the results. ${ }^{3}$

Non-invasive tests for determining the degree of liver fibrosis have now been developed, including the use of transient elastography such as Fibroscan and serum markers. Fibroscan can accurately determine the degree of liver fibrosis. Its limited availability and its requirement of the advanced device remain the disadvantages of this test.

Other non-invasive tests rely on the use of serum markers such as the APRI and FIB-4 indices under the WHO guidelines in 2015, especially for areas that do not have fibroscan facilities. Several research results show different results and remain controversial. Research on other serum markers using the GPR index has been developed to overcome this problem. The results of a study by Lemoine et al. showed that GPR can detect liver fibrosis more 
accurately than APRI and FIB-4. There have not been many studies on the correlation of APRI, FIB-4, and GPR with the HBeAg status of chronic hepatitis B patients. This study aimed to determine the correlation between APRI, FIB-4 and GPR with Fibroscan and HBeAg status of chronic hepatitis patients.

\section{METHODS}

This research was an analytic observational study with a cross-sectional design conducted at the Central Laboratory, Endoscopy and Hepatology Unit of Dr. Soetomo Hospital, Surabaya from June to September 2020. Inclusion criteria were patients with chronic hepatitis $\mathrm{B}(\mathrm{HBsAg}(+)>6$ months) aged $>18$ years with complete $\mathrm{HBeAg}$ results and $\mathrm{FO}-\mathrm{F} 4$ score of fibroscan and signed informed consent. Exclusion criteria were the coinfection of hepatitis $C$ and HIV, obesity, and a history of alcohol consumption. AST, ALT, and GGT levels were measured using the colorimetric enzymatic method with Dimension RXL, while platelets count was measured using the impedance method with Sysmex XN-1000. HBsAg test was carried out using magnetic particle chemiluminometric immunoassay method with ADVIA Centaur XP device. The results of the HBeAg test were obtained through medical records. The APRI, FIB-4, and GPR indexes were obtained from the calculation as follows:

Table 1. Characteristics of research subjects

$$
\begin{aligned}
& \text { APRI }=\frac{(\text { AST }(\mathrm{IU} / \mathrm{L}) / \text { AST (upper limit of normal level) }}{\text { Platelet count }\left(10^{9} / \mathrm{L}\right)} \times 100 \\
& \text { FIB-4 }=\frac{(\text { Age }(\text { years }) \times \text { AST }(\mathrm{IU} / \mathrm{L}))}{\left(\text { Platelet count }\left(10^{9} / \mathrm{L}\right) \times\left(\mathrm{ALT}(\mathrm{IU} / \mathrm{L})^{1 / 2}\right.\right.} \times 100 \\
& \text { GPR }=\frac{(\mathrm{GGT}(\mathrm{IU} / \mathrm{L}) / \mathrm{GGT} \text { (upper limit of normal level) }}{\text { Platelet count }\left(10^{9} / \mathrm{L}\right)} \times 100
\end{aligned}
$$

Determination of the degree of fibrosis using fibroscan showed the following results: F0-F1 (normal/mild): $<7.2 \mathrm{kPa}$, F2 (moderate): $7.2-<9.6 \mathrm{kPa}$, F3 (severe): $>9.6-12.5 \mathrm{kPa}$ dan F4 (cirrhosis): $>12.5 \mathrm{kPa}$.

Data were analyzed using the Spearman correlation test and $p<0.005$ was significant.

This study has received approval from the Health Research Ethics Committee of the Dr. Soetomo Hospital, Surabaya with number No: 1886/KEPK/III/2020.

\section{RESULTS AND DISCUSSIONS}

There were 50 research subjects who met the inclusion and exclusion criteria. The average age of the subject was $45.6 \pm 11.8$ years with the higher number of males (62\%), 47 subjects have received therapy, a higher number of negative (-) $\mathrm{HBeAg}$ status $(72 \%)$, and the fibroscan results showed a higher number of F0-F1 score (54\%). The AST level was 20-72 IU/L, ALT level was 21-140 IU/L, GGT level was 13-452 IU/L, and the mean of platelet count was $243.4 \pm 91.3 \times 10^{3} / \mu \mathrm{L}$ (Table 1 ).

\begin{tabular}{lc}
\hline Characteristics & Total (n=50) \\
\hline Age (years), mean \pm SD & $45.6 \pm 11.8$ \\
Gender, $\mathbf{n}(\%)$ & $31(62 \%)$ \\
$\quad$ Male & $19(38 \%)$ \\
Female & $56.7 \pm 4.6$ \\
Bodyweight (kg), mean \pm SD & $21.9 \pm 1.7$ \\
Therapy, $\mathbf{n}$ ), mean \pm SD & \\
Treated & 47 \\
Naïve & 3 \\
HBeAg, n (\%) & \\
Positive & $14(28 \%)$ \\
Negative & $36(72 \%)$ \\
Hb (g/dL), mean $\pm S D$ & $13.94 \pm 2.1$ \\
AST (IU/L), median (range) & $29(20-72)$ \\
ALT (IU/L), median (range) & $41.5(21-140)$ \\
GGT (IU/L), median (range) & $35(13-452)$ \\
PLT (10 $/ \mu L)$, mean $\pm S D$ & $243.4 \pm 91.3$ \\
Degree of fibrosis, n (\%) & \\
F0-1 & $26(54 \%)$ \\
F2 & $15(28 \%)$ \\
F3 & $1(4 \%)$ \\
& $8(14 \%)$ \\
\hline
\end{tabular}


There was no significant difference between APRI, FIB-4, and GPR indexes with the positive $(+)$ and negative (-) status of HBeAg (Table 2).

A significant correlation was found between the APRI, FIB-4, and GPR indices with fibroscan regardless of $\mathrm{HBeAg}$ status (Table 3 ). There was no significant correlation between APRI and GPR with fibroscan; however, there was a significant correlation between FIB-4 and fibroscan in chronic hepatitis B with (+) HBeAg status (Table 4). A significant correlation was found between the APRI, FIB-4, and GPR indices with Fibroscan in the research subjects with (-) HBeAg status (Table 5).

The mean age of the subjects in this study was $45.6 \pm 11.8$ years; however, a study by Adriati et al. found that the mean age of the research subjects was $39.7 \pm 11.8$ years in Makassar. ${ }^{4}$ A study by Dimzova et al. showed that the mean age of research subjects

Table 2. APRI, FIB-4, and GPR indices based on HBeAg status

\begin{tabular}{lccc}
\hline \multirow{2}{*}{ Characteristics } & \multicolumn{2}{c}{ HBeAg } & \multirow{2}{*}{$\mathbf{p}$} \\
\cline { 2 - 3 } & Positive $=16$ & Negative $=34$ & \\
\hline APRI, median (range) & $0.42 \pm 0.409$ & $0.47 \pm 0.540$ & 0.20 \\
FIB-4, median (range) & $0.62(0.34-9.72)$ & $0.83(0.24-10.82)$ & 0.58 \\
GPR, median (range) & $0.15(0.06-1)$ & $0.16(0.05-7.06)$ & 0.35 \\
Degree fibrosis, n(\%) & & & 0.31 \\
F0-1 & $8(50 \%)$ & $18(52.9 \%)$ & \\
F2 & $6(37.5 \%)$ & $9(26.5 \%)$ & \\
F3 & $0(0 \%)$ & $1(2.9 \%)$ & \\
F4 & $2(12.5 \%)$ & $6(17.6 \%)$ & \\
\hline
\end{tabular}

Table 3. Correlation between APRI, FIB-4, and GPR indices with Fibroscan

\begin{tabular}{llccc}
\hline Index & Fibroscan & $\mathbf{n = 5 0}$ & Correlation of Coefficient & $\mathbf{p}$ \\
\hline APRI & Mild (F0-1) & 26 & & \\
& Moderate (F2) & 15 & 0.454 & 0.001 \\
& Severe (F3) & 1 & & \\
& Cirrhosis (F4) & 8 & & \\
FIB-4 & Mild (F0-1) & 26 & 0.6 & \\
& Moderate (F2) & 15 & & \\
& Severe (F3) & 1 & & $<0.001$ \\
& Cirrhosis (F4) & 8 & & \\
GPR & Mild (F0-1) & 26 & 0.5 & \\
& Moderate (F2) & 15 & & \\
& Severe (F3) & 1 & & \\
& Cirrhosis (F4) & 8 & & \\
\hline
\end{tabular}

Table 4. Correlation between APRI, FIB-4, and GPR indices with fibroscan on positive (+) HBeAg status

\begin{tabular}{clccc}
\hline Index & Fibroscan & $\mathbf{n = 1 6}$ & Correlation of Coefficient & $\mathbf{p}$ \\
\hline APRI & Mild (F0-1) & 8 & & \\
& Moderate (F2) & 6 & 0.295 & 0.267 \\
& Severe (F3) & 0 & & \\
& Cirrhosis (F4) & 2 & & 0.049 \\
FIB-4 & Mild (F0-1) & 8 & & \\
& Moderate (F2) & 6 & & \\
& Severe (F3) & 0 & & 0.1499 \\
& Cirrhosis (F4) & 2 & & \\
\hline
\end{tabular}


Table 5. Correlation between APRI, FIB-4, and GPR indices with fibroscan on negative (-) HBeAg status

\begin{tabular}{clccc}
\hline Index & Fibroscan & $\mathbf{n = 1 6}$ & Correlation of Coefficient & $\mathbf{p}$ \\
\hline APRI & Mild (F0-1) & 18 & & \\
& Moderate (F2) & 9 & 0.486 & 0.004 \\
& Severe (F3) & 1 & & \\
& Cirrhosis (F4) & 6 & & \\
FIB-4 & Mild (F0-1) & 8 & & \\
& Moderate (F2) & 6 & 0.648 & \\
& Severe (F3) & 0 & & $<0.001$ \\
& Cirrhosis (F4) & 2 & & \\
\hline
\end{tabular}

was $38.8 \pm 10.7$ years in the Republic of Macedonia. ${ }^{5}$ Age in this study was similar to that of the previous study. The rate of progression of acute to chronic hepatitis $B$ is mainly determined by the age at the time of infection. Hepatitis B infection at a younger age is more likely to develop into chronic hepatitis $B$. The progression rate of chronic hepatitis $B$ with acquired perinatal infection is nearly $90 \%, 20-50 \%$ for infections acquired at 1-5 years of age, and $<5 \%$ for infections acquired during adulthood. ${ }^{1}$

Most of the subjects of this study were male (62\%), in accordance with a study by Adrianti et al. research, which found a higher number $(66 \%)$ of male subjects. ${ }^{4}$ In addition, a study by Dimzova et al. found $74 \%$ male research subjects and a study by $\mathrm{Li}$ et al. found $65.3 \%$ male research subjects in China, indicating similar results to this study. ${ }^{5,6}$ References suggest that male have more risk factors for hepatitis B, C, and hepatocellular carcinoma. Hepatocellular carcinoma due to hepatitis $B$ is more common in male with a male to female ratio of $1: 4-1: 7$. $^{7}$

This study found no subjects with obesity, which can affect GGT levels and Fibroscan results in this study. ${ }^{8.9}$ This study was similar to that of Li et al., which involved research subjects with $\mathrm{BMI}<28 \mathrm{~kg} / \mathrm{m}^{2}$. $^{10}$

Almost all patients in this study had received antiviral therapy, following a study by Li et al. in Shanghai which found that all study subjects had been treated. However, this study was different from the research of Liu et al. and Li et al. in Shanghai. ${ }^{6,11}$ Recent studies have shown that antiviral administration can suppress the hepatitis $B$ virus and reduce necroinflammatory activity and improve the degree of fibrosis. Previous studies have shown that FIB-4 was unreliable for detecting fibrosis regression after administration of antiviral therapy. Another study showed that APRI was not correlated with regression or progression of fibrosis in hepatitis B patients treated with Tenofovir. ${ }^{12}$

Elevated AST levels indicate liver cell destruction that triggers the release of AST from mitochondria and liver cell cytoplasm and reduced clearance due to Bishop's liver fibrosis. ALT levels are a major marker of liver cell inflammation and are specific for the cytoplasm of liver cells. ${ }^{13} \mathrm{An}$ increase in ALT levels is a host immune response against hepatocytes infected with the hepatitis B virus. The higher ALT levels indicate greater hepatocyte damage due to increased immune response. ${ }^{14}$ The GGT enzyme is considered an important parameter to predict the severity of liver fibrosis and is present in hepatocytes and biliary tract cells. Elevated GGT levels indicate damage to hepatocytes due to liver fibrosis. The GPR index will increase according to the increase in the degree of fibrosis. GGT enzymes are generally not elevated in mild chronic hepatitis and inactive cirrhosis. GGT levels increase $>2$ times the upper limit of the normal level in pre-cirrhotic states. ${ }^{8}$ Several studies have stated that the cause of thrombocytopenia in liver disease is due to splenomegaly, hypersplenism, portal hypertension, and decreased production of thrombopoietin. A recent study reported an increased platelet count in chronic hepatitis B patients with significant degrees of fibrosis who had received antiviral therapy showed improvement in liver fibrosis. ${ }^{15}$

There was no significant difference between the APRI, FIB-4, and GPR indices in chronic hepatitis B with positive (+) or negative (-) HBeAg. The APRI, FIB-4, and GPR indices were higher depending on the severity of fibrosis. World Health Organization announced APRI and FIB-4 in 2015 as parameters to detect liver cirrhosis in areas with limited resources. ${ }^{16}$ The APRI and FIB-4 indices have moderate accuracy 
and sensitivity in chronic hepatitis B-induced fibrosis. ${ }^{5}$ Lemoine et al. proposed GPR as a more accurate laboratory marker compared to APRI and FIB-4 to determine the degree of fibrosis in chronic hepatitis B patients in West Africa in June 2015. ${ }^{16}$

The results of the Spearman correlation test showed that there was a significant correlation between APRI, FIB-4, and GPR with fibroscan, indicating that the APRI and FIB-4 indices will increase along with the increasing degree of fibrosis.

The APRI, FIB-4, and GPR indices showed a positive correlation with the degree of Metavir fibrosis $(r=0.532, p<0.001$ and $r=0.459, p<0.001$; $r=0.475, p<0.001)$. APRI, FIB-4, and GPR indices tend to increase with an increasing degree of liver fibrosis. A study by Wang et al. showed that APRI and FIB-4 indices were positively correlated with liver biopsy in determining the degree of fibrosis $((r=0.363$, $p<0.001, r=0.447, p<0.001$ and $r=0.439, p<0.001$, respectively). ${ }^{17}$ Research by Huang et al. showed a significant correlation between APRI, FIB-4, and GPR indices with fibroscan in identifying the degree of significant fibrosis, severe fibrosis, and liver cirrhosis. ${ }^{18}$ Research by Lee et al. showed that APRI, FIB-4, and GPR indices were significantly correlated with fibroscan. $(r=0.72, p<0.001 ; r=0.68, p<0.001$; $r=0.68, p<0.001$, respectively) in chronic hepatitis $B$ patients and can be used as a marker of the severity of fibrosis. ${ }^{19}$ Research by Hu et al. showed that APRI, FIB-4, and GPR indices were positively correlated with the degree of liver fibrosis on liver biopsy $p<$ $0.0120 .{ }^{20} \mathrm{~A}$ study by Ren et al. study showed that GPR is a simple serum marker and can predict the presence of fibrosis. ${ }^{21} \mathrm{~A}$ study by Lian et al. showed that GPR has diagnostic accuracy in predicting moderate fibrosis, severe fibrosis and cirrhosis. ${ }^{22}$

The results of this study were different from those of Kim et al. and Li et al. A study by Kim et al. showed that APRI and FIB-4 indices were not correlated with liver biopsy in chronic hepatitis B patients who had been treated for 240 weeks. This was due to the differences in subjects and research methods. This study involved research subjects with an average history of therapy less than 4 years and used fibroscan as the test method. ${ }^{12} \mathrm{~A}$ study by Li et al. showed that APRI and FIB-4 had a weak correlation with liver biopsy in determining the degree of fibrosis in chronic hepatitis $B$ patients $(r=0.16$, $p<0.012$ and $r=0.27, p<0.001)$. This was due to different test methods and the use of more selective inclusion and exclusion criteria compared to this study. ${ }^{6}$ The study by $\mathrm{Li}$ et al. involved research subjects who had not received antiviral therapy with ALT levels 2 times the upper limit of normal level and used liver biopsy as the method to determine the degree of fibrosis.

A significant correlation was found between the APRI, FIB-4, and GPR indices with Fibroscan in chronic hepatitis B patients with negative (-) $\mathrm{HBeAg}$ status. There was no significant correlation between APRI and GPR indices with Fibroscan; however, there was a significant correlation between FIB-4 and Fibroscan indices in chronic hepatitis B with positive (+) HBeAg status. In contrast to the study by Liu et al., which showed moderate accuracy values of the APRI index, FIB-4 and GPR indices were able to predict the degree of fibrosis in chronic hepatitis $B$ with positive $(+)$ and negative (-) $\mathrm{HBeAg}$.

The progression of fibrosis in chronic hepatitis $B$ may differ between positive $(+)$ and negative (-) HBeAg. Chronic hepatitis B patients with positive (+) $\mathrm{HBeAg}$ and normal ALT levels showed mild or no fibrosis. Contrastingly, moderate-severe fibrosis was found in those with elevated ALT levels. Chronic hepatitis B patients with negative (-) $\mathrm{HBeAg}$ with normal ALT levels were in the inactive phase, which showed improvement of liver cell injury and fibrosis regression, whereas those with elevated ALT levels showed significant recurrent liver injury with recurrent progressive fibrosis. ${ }^{23} \mathrm{HBeAg}$ triggers the activation and proliferation of hepatic stellate cells, which play an important role in the process of liver fibrosis. Positive (+) HBeAg indicates the severity of liver inflammation in chronic hepatitis B. ${ }^{24}$ This study found no correlation between serum markers on positive (+) HBeAg and the degree of fibrosis based on fibroscan. This might be due to normal levels of AST, ALT, and GGT that will affect the results of the index. In this situation, it was necessary to perform other tests to determine the degree of fibrosis such as fibroscan for therapeutic purposes.

\section{CONCLUSIONS AND SUGGESTIONS}

Similar to APRI and FIB-4 indices, the GPR index can be used as another serum marker to determine the presence of liver fibrosis, especially in areas without fibroscan facilities. The APRI, FIB-4, and GPR indices were unable to detect the presence of fibrosis in chronic hepatitis B with positive (+) HBeAg in this study. It was necessary to include the HBV DNA test, which might affect the correlation results.

\section{REFERENCES}

1. Seto WK, Lo YR, Pawlotsky JM, Yuen MF. Chronic hepatitis B virus infection. Lancet, 2018; 392(10161): 2313-24.

2. Tang LSY, Covert E, Wilson E, Kottilil S. Chronic hepatitis B infection a review. JAMA-J Am Med Assoc, 
2018; 319(17): 1802-13.

3. Mauss S, Berg T, Rockstroh J, Sarrazin C, Wedemeyer H. Hepatology-a clinical textbook. 9th Ed., Germany, Flying Publisher, 2018; 423.

4. Adrianti E, Kurniawan BL, A. Samad I. Comparisons of fibro $Q$ index and FIB-4 in various stages of chronic $B$ hepatitis. Indones J Clin Pathol Med Lab, 2018; 25(1): 68.

5. Dimzova M, Kondova-Topuzovska I, Bosilkovski M, Ivanovski L, Milenkovic Z, et al. Non-invasive biomarkers in assessment of liver fibrosis in patients with HBeag negative chronic hepatitis B. Open Access Maced J Med Sci, 2018; 6(6): 1052-8.

6. Li Q, Ren X, Lu C, Li W, Huang Y, Chen L. Evaluation of APRI and FIB-4 for noninvasive assessment of significant fibrosis and cirrhosis in $\mathrm{HBeAg}$-negative CHB patients with ALT?2 ULN A retrospective cohort study. Med (United States), 2017; 96(12): 1-7.

7. Ruggieri A, Gagliardi MC, Anticoli S. Sex-dependent outcome of hepatitis B and C Viruses infections: Synergy of sex hormones and immune responses? Front Immunol, 2018; 9: 1-7.

8. Wang RQ, Zhang QS, Zhao SX, Niu XM, Du JH, et al. Gamma-glutamyl transpeptidase to platelet ratio index is a good non-invasive biomarker for predicting liver fibrosis in Chinese chronic hepatitis B patients. J Int Med Res, 2016; 44(6): 1302-13.

9. De Lédinghen $V$, Vergniol J. Transient elastography for the diagnosis of liver fibrosis. Expert Rev Med Devices, 2010; 7(6): 811-23.

10. Li Q, Chen L, Zhou Y. Diagnostic accuracy of liver stiffness measurement in chronic hepatitis B patients with normal or mildly elevated alanine transaminase levels. Sci Rep [Internet]. 2018; 8(1): 1-7. Available from: http://dx.doi.org/10.1038/s41598-018-23646-2 (accessed at 5 June, 2020).

11. Liu D, Zhang Z, Wang $Y$, Ding $R$, Zhou $X$, et al. Comparative evaluation of GPR versus APRI and FIB-4 in predicting different levels of liver fibrosis of chronic hepatitis B. J Viral Hepatol, 2018; 25(5): 581-9.

12. Kim WR, Berg $T$, Asselah $T$, Flisiak $R$, Fung $S$, et al. Evaluation of APRI and FIB-4 scoring systems for non-invasive assessment of hepatic fibrosis in chronic hepatitis B patients. J Hepatol [Internet]. 2015. Available from: http://dx.doi.org/10.1016/ j.jhep.2015.11.012 (accessed at 5 June, 2020).

13. Bishop ML, Fody EP, Schoeff LE. Clinical chemistry -principles, techniques and correlations. $8^{\text {th }}$ Ed., Philadelphia, Wolters Kluwer, 2018; 721-722.
14. Wu JP, Mao WL. Review of serum biomarkers and models derived from them in HBV-related liver diseases. Dis Markers, 2020; 2020.

15. Yang $Y$, Wang L, Yan L, Zhang L, Zhou W, et al. Platelet count is closely associated with the severity of liver injury in patients with chronic hepatitis B virus infection: A cross-sectional study. Exp Ther Med, 2020; 20(1): 243-50.

16. Wu JF, Chang MH. Natural history of chronic hepatitis $B$ virus infection from infancy to adult life-the mechanism of inflammation triggering and long-term impacts. J Biomed Sci, 2015; 22(1): 1-7.

17. Wang J, Yan $X$, Yang $Y$, Chang $H$, Jia $B$, et al. A novel predictive model using routinely clinical parameters to predict liver fibrosis in patients with chronic hepatitis B. Oncotarget, 2017; 8(35): 59257-67.

18. Huang R, Wang G, Tian C, Liu Y, Jia B, et al. Gamma-glutamyl-transpeptidase to platelet ratio is not superior to APRI, FIB-4 and RPR for diagnosing liver fibrosis in $\mathrm{CHB}$ patients in China. Sci Rep [Internet]. 2017; 7(1): 1-10. Available from: http://dx.doi.org/10.1038/s41598-017-09234-w (accessed at 5 June, 2020).

19. Lee J, Kim MY, Kang SH, Kim J, Uh Y, et al. The gamma-glutamyl transferase to platelet ratio and the FIB-4 score are noninvasive markers to determine the severity of liver fibrosis in chronic hepatitis B infection. Br J Biomed Sci, 2018; 75(3): 128-32.

20. Hu YC, Liu H, Liu XY, Ma LN, Guan YH, et al. Value of gamma-glutamyltranspeptidase-to-platelet ratio in diagnosis of hepatic fibrosis in patients with chronic hepatitis B. World J Gastroenterol, 2017; 23(41): 7425-32.

21. Ren T, Wang H, Wu R, Niu J. Gamma-glutamyl transpeptidase-to-platelet ratio predicts significant liver fibrosis of chronic hepatitis B patients in China. Gastroenterol Res Pract, 2017; 1-7.

22. Lian MJ, Zhang JQ, Chen SD, Zhang DD, Yang YY, Hong GL. Diagnostic accuracy of Y-glutamyl transpeptidase-to-platelet ratio for predicting hepatitis B-related fibrosis: A meta-analysis. Eur J Gastroenterol Hepatol, 2019; 31(5): 599-606.

23. EASL. EASL 2017 clinical practice guidelines on the management of hepatitis B virus infection. J Hepatol, 2017; 67: 370-98.

24. Chen Y, Li Y, Li N, Fan X, Li C, et al. A non-invasive score to predict liver fibrosis in $\mathrm{HBeAg}$-positive hepatitis $\mathrm{B}$ patients with normal or minimally elevated alanine aminotransferase levels. Dis Markers, 2018; 2018. 